\title{
The Effects of Displacement Induced by Thermal Perturbations on the Structure and Stability of Boundary-Layer Flows ${ }^{1}$
}

\author{
C. Treviño
}

A. Luiñàn

\begin{abstract}
The free-interaction influence of a thermal expansion process in boundary-layer gas fow is analyzed using the formalism of triple-deck theory. The plyssical model considered is the forced convection of a gas flowing over a flat plate subject to a heated slab. Both linearized and full nonlineal" solutions are obtaincd using fourier transform molhods and spectral numerical technigues. The influence of monochromatic thermal perturbation on boundary-layer stability (lower branch) is studicd and first-order correction of the lower branch nental stability curve for the boundary-layer fow has been obtained. The shift of neutral stability is then computed for different values of the themal perturbation wave number, making unstable some olherwise stable modes.
\end{abstract}

\section{Introduction}

In a gaseous boundary-layer fow the expansion and displacement effects due to surface thermal distrubances can be very important and therefore can modify the flow structure and stability. ln particular, if the surface lemperature changes abruptly in a small region, the velocity and pressure gradients generated can be very strong and the effects have 10 be retained 10 leading order in boundary-layer analysis. 'These pressure gradients are generated by interaction with the outer potential fow and have an important influence on the flud close to the wall where the convective terms are small. The triple-deck structure can be used to explain these lypes of flows correctly and to predict boundary-layer separation. Gas expansion duc to heating or cooling is produced in a viseous nonisothermal lower deck and is impressed as displacement effects, thus generating pressure gradicnts, in the upper deck. The triple-deck concept has been introduced in the classical works by Stewartson and Williams [1], Messiter [2], and Stewartson [3] studying the influence on the drag coeflicient of a finitc-length flat plate, using a threc-layer structure. A comprehensive review on this subject is given by Smith [4], where the sane structural argument of triple-deck theory can be applied immediately to many other incompressible and compressible problems. The etrects of surface temperature changes in a vertical fat plate on the free convective flow structure were analyzed by Messiter 
and Liñan [.5']. Due to the lack of an upper-deck flow, the analysis can be carried out by a two-laycr concept, where the resulting cquations for the lower viscous and heal-conducting sublayer, are linear. However, when describing the eflect of surface clange in temperature on a forced boundary-layer flow, triple-deck analysis is then rejuired. Recently. Méndez ef al. [6] studied the fuid mechanical structure of a gascous forced boundary-layer flow produced by the thermal expansion process using triple-deck theory. They analyzed the case of a step-change on surface temperature. The effects of the displacoment, due to gas cxpansion resulting from the sudden temperature risc, increase strongly when increasing the temperature ratio, defined by the ratio of the maximum plate temperature to the fluid temperature. For a critical value of this parameter, flow separation, ahead of the poin where the surface temperature changes, can be reached. This critical value is close $10,3 P^{1 / 3}$ for a perfect gas. $P_{r}$ is the Prandt number which relates viscous with thermal diffusivity. Fluids with a large Prandil number make the expansion process weaker and lhus a larger temperalure ratio is needed to obtain flow separation. The effects of thermal expansion are felt in a region, around the point where the temperature changes, of size (related to the boundary-layer thickness $h^{*}$ ) of the order of $R_{h}^{1,4}$, where $R_{h}$ is the Reynolds number based on the boundary-layer thick ness.

The changes in the flow structure due to thermal perturbalions have a strong influence on the stability of the boundary-layer flow. In rejation to flow stability, the triple-deck structure is appropriate in the lower branch of the neutral stability curve for large Reynolds numbers. In this limit, thercfore, full interaction with the outer potential flow' must be considered [7]. Linear and weakly nonlinear stability analyses were carrice out by Sinith [7] [9], where nonparallel effects were also included. He obaincd a dispersion relation in tcrms of the Airy function and solved it numerically. Goldstein [10] and Goldstein and Hultgren [11] developed an analysis to study the coupling between a small but sudden variation in surface geonctry and small amplitude acoustic waves. The objective was to explain the receptivity measurements calried out by Leelacy and Shapiro [12], who oblained a high value of the coupling coefficient. Bodonyi ef al. [13] numerically studied this receptivity problem but with finite size surface variations.

Surface cooling effects on compressible boundary-layer llows have been addressed by several works [14]-[16]. Seddougui et al. [14] sudied the influence of wall cooling on boundary-layer instability. They found that moderate surface cooling can enhance the Tollmien -Schlichting viseous mode aclivity, where the spatial growth of viscous modes can be comparable with inviscid ones. They also found that cooling can destabilize otherwise stable viscous and inviscid modes at any Mach number. They obtaned good agreement with the experimental results of Lysenko and Maslov [15]. On the other hand, strong wall cooling in a hypersonic flow has been analyzed by Brown et al. [16]. They conchded, from the numerical solutions of the triple-deck in the weak global interaction regime, that separation and reattachment on a compressive ramp cannot be effectively eliminaled or delayed by lowering the wall temperature, but as the wall temperature is reduced the triple-deck dimension decreases drastically and, therefore, the upstream influence. In all the works a uniform wall temperature was assumed.

There are many interesting low Mach number flows with strong temperature gradients which can be analyzed using the franework of the triple-deck concept. There is experimental cvidence that premixed flames are stabilized by acrodynamic effects such as low local velocities and even flow recirculation that would not exist without the presence of the flame. These recirculating llows are generated by pressure gradients that are induced by the interaction of the flame with the inviscid flow producing the separation of the boundary layer [177. In diffusion flame experiments in boundary layers with injection, pressure gradients have been predicted from the measured velocity field in the vicinity of the flame leading edge [18] The existence of low-and higl?-pressure regions suggests the possibility of using higher-order effects in the boundary-layer approximation that influences the structure and showing the interactions between the flame and flow field. In particular, higher-order effects are very important in processes with density variations. In this case the expansion of the gas flowing along the body changes in an important way the displacement thickness, producing a strong interaction with the potential flow. Higher-order effects in combustion processes werc considered in several works [19], [20]. There is also interest in the study of reacting flows over catalytic surfaces. In this case the surface temperaturc can increase in a short distance depending on the sizes of the active sites on the catalytic wall.

In this work we use spectral numerical techniques to describe the strong surface thermal perturbation in a region assumed to be of size of the order of the triple-deck. "Therefore, the problem can be sealed using the classical triple deck scaling laws, as shown for the case of a step change in surface temperature [6]. In the analysis we treat in detail the ease (fo: simplicity) when the surface temperature is equal to the ambient 
temperature, except in the triple-deck region of the order of $R_{h}^{1 / 4}$ times the boundary-layer thickness, where the temperature can assume any arbitrary form. Also for simplicity we assume an ideal gas with a unity Chapman-Rubesin parancter (viscosity coeficiont lincarly dependent on tempeature). The changes in pressure incluced by gas expansion and felt on the upper deck as displacement effects, are assumed to be very' strong in comparison with that of the frec stram in the unperturbed fow. If we increase the temperature, an overpressure \%one appears in the upstream region followed by an underpressure zone downstream. Therefore, there are two zones with adverse pressure gradients and thus two regions where the shear stresses at the wall (skin friction) are lower than the initial values. The region in between presents a strong peak in positive skin friction. On the other hand, if the temperature in the triple-deck region is smaller than the ambient one (cooling), the eflects are reversed with a single region with adverse pressure gradients resulting in a strong reduction in minimum skin friction. In Section 2 we present the nondimensional triple-deck governing equations, retaining the unsteady terms for use in the stability analysis, assuming that the characteristic time of change is of the order of the residence time in the triple-deck region. In Section 3 we present the analysis of the linearized response of the boundary layer under small perturbations in the surface temperaturc, obtaining the results by using Fouricr transform techniques. Nonlinear analysis is given in Scction 4, using numerical methods based on spectral tcchniques. We assume here the case for a smooth surfacc-temperature distribution. $\Lambda$ stability analysis is presented in Section 5 by assuming a monochromatic surface-temperature perturbation. We are interested in the shift of the neutral stability curve when surface thermal perturbations are present in a gaseous boundary-layer fow. Finally, concluding remarks are presented in Section 6.

\section{Governing Equations}

The physical model analyzed is the following. We consider a gaseous boundary-layer flow past a body of arbitrary shape, as shown in Figure 1. The wall temperalure is assumed for simplicity to be the same as the frec-stream temperaturc, $T_{w}^{*}$, except in a small region of order $h^{*} R_{h}^{1 / 4}$ around a point $\left(x^{*}=x_{0}^{*}, y^{*}=0\right)$, where the boundary-layer thick ness is $h^{*}$ and the free-stream velocily is $u^{*}$. Here, $R_{h}$, denotes the Reynolds

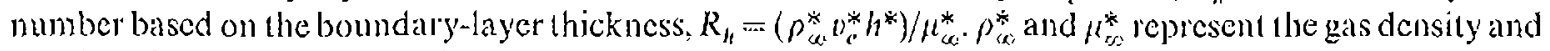
the viscosity coefficient in the free strcam, respectively. Assuming the healted/cooled region to be confined in a stream wise length of order $h^{*} R_{h}^{1 / 4}$, the free interaction phenomena can be described by the triple-deck flow structure. The wall-temperature difference in this region, $T_{v}^{*}-\Upsilon_{w}^{*}$, can take any arbitrary form, tending to \%cro at both ends of the heated/cooled triple-deck region. The evolution of the displacement thickness changes in an important way due to the expansion $\left(T_{w}^{*}>T_{\omega}^{*}\right)$ or compression $\left(T_{*}^{*}<T_{c}^{*}\right.$ ) process, causing retention of the pressure graclients in the leading-order governing equations. The triple-deck structure can be described by three layers (upper, main, and lower deck), cach salisfying appropriated governing equations [4]. For this probiem, the viscous and nonisothermal cffects are concentrated in the lower deck,

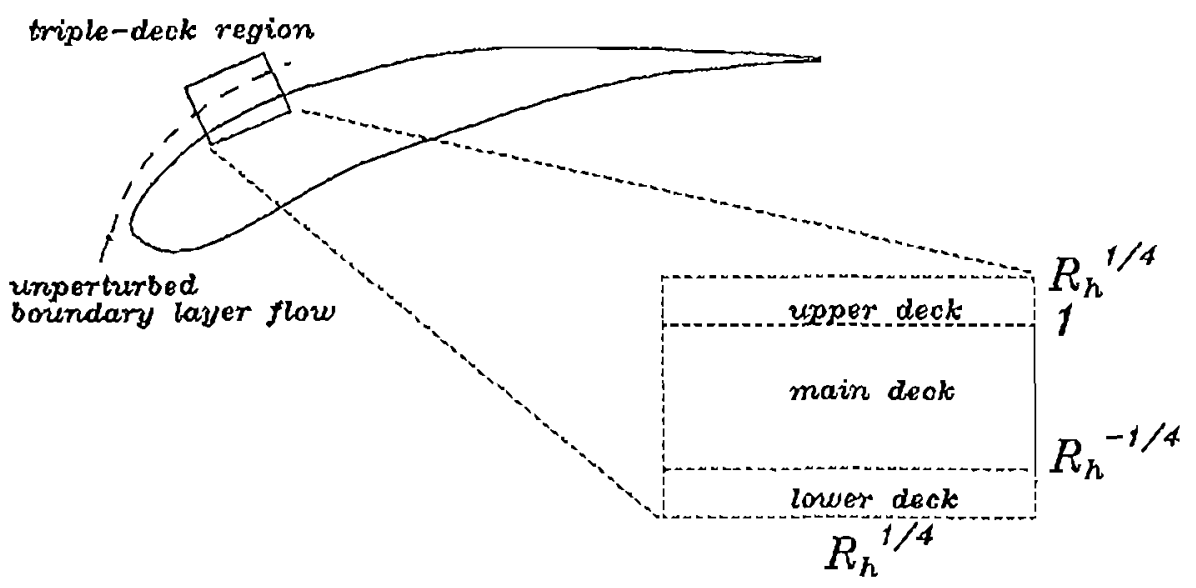

Fïgure 1. Schenatical diagram of the interaction problem. 
while the flow in the main and upper decks is incompressible and inviscid. The gas expansion generated in the lower deck produces, after being transmitted through the main deck, displacement effects in the upper deck. The governing equations in the isothermal upper deck reduce to the well-known pressure-displacement relation [3], to be given later. The inain deck transmits the pressure gadicnts down to the lower deck. ln order to consider those regions where the the mal expansion/compression becomes considerable, it is necessary to introduce adequate triple-deck structure scaling. The nondimensional unsteady governing equations in the lower deck, assuming the Eckert number to be completely negligible (low Mach number flows), are given by

$$
\begin{gathered}
\frac{\partial p}{\partial \tilde{T}}+\frac{\partial(\rho \tilde{u})}{\partial \tilde{x}}+\frac{\partial(\rho \tilde{\theta})}{\partial \tilde{y}}=0, \\
\rho \frac{\partial \tilde{u}}{\partial \tilde{t}}+\rho \tilde{u}-\frac{\partial \tilde{u}}{\partial \tilde{x}}+\rho \tilde{\partial} \frac{\partial \tilde{v}}{\partial \tilde{y}}=-\frac{\partial \tilde{p}}{\partial \tilde{x}}+\frac{\partial}{\partial \tilde{y}}\left(\mu \frac{\partial \tilde{u}}{\partial \tilde{y}}\right), \\
\frac{\partial p}{\partial y}=O\left(R_{h}{ }^{-1}\right), \\
\rho \frac{\partial T}{\partial \tilde{T}}+\rho \tilde{u} \frac{\partial T}{\partial \tilde{x}}+\rho \tilde{v} \frac{\partial T}{\partial \tilde{y}}=\frac{1}{P r} \frac{\partial}{\partial \tilde{y}}\left(\mu \frac{\partial T}{\partial \tilde{y}}\right),
\end{gathered}
$$

where the nondimensional variables are defined by

$$
\begin{aligned}
& \tilde{x}=\frac{\left(x^{*} \cdots x_{0}^{*}\right)}{h^{*} R_{h}^{1 / 4}}, \quad \tilde{y}=\frac{y^{*} R_{h}^{1 / 4}}{h^{*}}, \quad \tilde{u}=\frac{u^{*} R_{h}^{1 / 4}}{v_{t}^{*}}, \quad \tilde{v}=\frac{v^{*} R_{h}^{3 / 4}}{v_{\mathrm{e}}^{*}},
\end{aligned}
$$

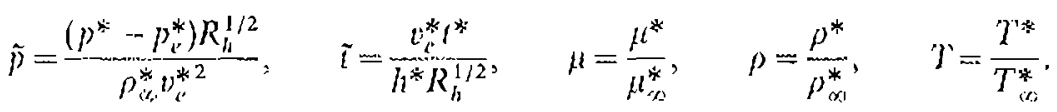

In the above equations $x^{*}$ and $y^{*}$ correspond to the longitudinal and normal coordinates, respectively; $u^{*}$ and $v^{*}$ correspond to the fluid velocily components in the $x^{*}$ and $y^{*}$ dircetions, respectively; $\eta^{*}$ is (lie pressure and $p_{*}^{*}$ is the pressure of the unperturbed now at the position $x^{*}=x_{0}^{*} ; t^{*}$ is the time. The boundary-layer thickness $h^{*}$ is defined in such a way that it makes the velocity gradient at the wall of the unperturbed flow equal to $v_{k}^{*} / h^{*}$; Pr represents the Prandt number defined by $P r=\mu_{\alpha:}^{*} C_{p}^{*} / k_{\alpha}^{*}$, where $C_{p}^{*}$ is the specific heat at constant pressure and $k_{\omega}^{*}$ represents the heat conductivity coefficient of the gas. We also assume that the Prandil number is constant. In addition to the above equations, we need an equation of state. In this case, for simplicity, we use an ideal gas law given by $p^{*}=p_{c}^{*} \rho T$, with a gas viscosity coefficient linearly dependent on temperature (Chapman-Rubesin paramcter, $C=\mu p=1$ ). For low Mach number flows. the pressure variations are small compared with the ambient pressure and therefore the pressure can be assumed to be constant only for the state equation. It can be written in nondimensional form as

$$
\rho T=1 \text {. }
$$

The boundary conditions reguired to complete the problem statement are specified by the following relationships: at $\tilde{y}=0$,

$$
\tilde{u}=\tilde{u}=0, \quad T=T_{\mathrm{v}}(\tilde{x}), \quad \text { with } \quad T \rightarrow 1 \text { for } \quad|\tilde{x}| \rightarrow \infty .
$$

$\Lambda s|\tilde{x}| \rightarrow \infty$, the solution must malch the unperturbed fiow solution given by $\tilde{u} \rightarrow \tilde{y}$ and $T \rightarrow 1$. For large values of $\bar{s}$, the solution must match that of the main deck. We do not go into details that can be found elsewhere [3]. The corresponding boundary conditions for large $\tilde{y}$ are then given by

$$
\tilde{u} \sim \tilde{y}+\tilde{A}(\tilde{x}, \tilde{l}) \text { for } \tilde{y} \rightarrow \infty \text {. }
$$

Herc $-\tilde{A}(\tilde{x}, \tilde{i})$ represents the displacement thickness and also the velocity slip at the base of the main deck, corresponding to the inviscid perturbation of the upstream solution by the induced pressure gradient. In addition, a boundary condition for the temperature is required in order to complete the match with the main-deck solution and is given by

$$
T \rightarrow 1 \quad \text { for } \quad \tilde{y} \rightarrow \infty
$$


Finally, an interaction condition, which is derived by thin airfoil linearized theory, is required to match the upper-deck solution and it corresponds to the well-known pressure-displacement relation [3]

$$
\tilde{p}(\tilde{x}, \tilde{i})=\frac{1}{\pi} \int_{\ldots=}^{\infty} \frac{\hat{u} \tilde{A}\left(\tilde{x}_{1}, \tilde{i}\right)}{\partial \tilde{x}_{1}} \frac{d \tilde{x}_{1}}{\left(\tilde{x}-\tilde{x}_{1}\right)} .
$$

In the last equation, the integral represents the principal value of the Cauchy-Hibert integral. Clearly, for fixed values of $R_{h}$ and $P r$, the solution depends on the magnitude and distribution of $T_{\text {I. }}(\tilde{x})$. The above set of equations defines the thermal expansion/compression problem within the longitudinal scale $\left(x^{*}-x_{0}^{*}\right) \sim h^{*} R_{h}^{1 / 4}$. However, for the $P r$ and $\mu p$ constants, these equations can be simplified cven more by cmploying the Howarth - Dorodnitynn thansformation [21], giving an equivalent set of quasi-incompressible governing equations. By introducing the following nondimensional variables,

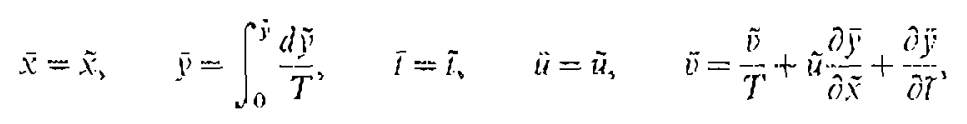

we obtain the corresponding lower-deck nondimensional governing equations:

$$
\begin{aligned}
& \frac{\partial \bar{u}}{\partial \bar{x}}+\frac{\partial \hat{v}}{\partial \hat{j}}=0, \\
& \frac{\partial \bar{u}}{\partial t}+\bar{u} \frac{\partial \bar{u}}{\partial \dot{x}}+\bar{p} \frac{\partial \bar{u}}{\partial \bar{y}}=-T \frac{\partial \tilde{p}}{d \bar{x}}+\frac{\partial^{2} \bar{u}}{\partial \bar{y}^{2}}, \\
& \frac{\partial T}{\partial T}+\frac{\partial T}{\partial \bar{x}}+\frac{\partial T}{\partial y}=\frac{1}{P r} \frac{\partial^{2} T}{\partial \bar{y}^{2}} .
\end{aligned}
$$

Equations (12)-(14) have to be solved with the following boundary conditions,

$$
\begin{gathered}
\bar{u}=\bar{u}=0, \quad T=T_{w}(\bar{x}) \quad \text { al } \quad \bar{y}=0, \\
\bar{u} \rightarrow \bar{y}, \quad T \rightarrow 1 \quad \text { for } \quad|\bar{x}| \rightarrow \infty, \\
\tilde{u} \rightarrow \bar{y}+\bar{A}(\bar{x}, \bar{i})+\int_{0}^{u}(T-1) d \bar{y} \quad \text { ror } y \rightarrow \infty,
\end{gathered}
$$

and the appropriate initial conditions. The nonconstant nondimensional density, $\rho=1 / T$, does not fully disappear from the governing equations, but remains only in the pressure-gradient term in the momentum equation (13). The lower-deck equations are completed with the intcraction relationship

$$
\tilde{p}(\bar{x}, \bar{l})=\frac{1}{\pi} \int_{-\infty}^{\infty} \frac{\partial \tilde{A}\left(\ddot{x}_{1}, \bar{l}\right)}{\partial \tilde{x}_{1}} \frac{d \tilde{x}_{1}}{\left(\bar{x}-\ddot{x}_{1}\right)} .
$$

Using the now lower-deck normal coordinate $\ddot{y}$ allows us to separate the displacement eflects denoted by $\tilde{A}\left(\hat{x}_{1}, \bar{l}\right)$ and the gas-expansion effects denoted by the inlegral term $\int_{0}^{(x)}(T-1) d \bar{l}$. In the next two sections we analyze both the linear and nonlinear steady frows induced by a heated/coolcd slab.

\section{Linearized Theory For $T_{w} \rightarrow 1$}

Equations (12) (16) can be solved analytically by assuming that the surface-temperature changes are very small compared with the ambient temperature. Defining a small parameter $\delta$ as the maximum nondimensional temperature difference, $\delta=\max \left(T_{w}-1\right)$, the lower-deck governing equations can be lincarized around the undisturbed boundary-layer profile, by expanding the flow variables as follow:

$$
\begin{gathered}
u=\bar{y}+\hat{u}=\bar{y}+\delta \hat{u}_{0}+\cdots, \quad \bar{v}=\delta \hat{o}_{0}+\cdots, \\
\bar{p}=\delta \hat{p}_{0}+\cdots, \quad \tilde{A}=\delta \hat{A}_{0}+\cdots, \\
T=1+\hat{T}=1+\delta \hat{T}_{0}+\cdots, \quad \tau=1+\hat{\tau}=1+\delta \hat{\tau}_{0}+\cdots,
\end{gathered}
$$

where $\tau$ corresponds to the nondimensional skin friction defined by $\tau=d \bar{u} / d \bar{y}$, which is exactly unity for the unperturbed isothermal boundary-layer flow. Therefore, the linear nondimensional governing equations 
can be reduced, up to first order, to

$$
\begin{array}{r}
\frac{\partial \hat{u}_{0}}{\partial \bar{x}}+\frac{\partial \hat{v}_{0}}{\partial \tilde{y}}=0, \\
j \frac{\partial \hat{\tau}_{0}}{\partial \bar{x}}-\frac{\partial^{2} \hat{\tau}_{0}}{\partial \tilde{y}^{2}}=0, \\
\bar{y} \frac{\partial \hat{T}_{0}}{\partial \bar{x}}-\frac{1}{P r} \frac{\partial^{2} \hat{\tau}_{0}}{\partial j^{2}}=0,
\end{array}
$$

and

$$
\begin{aligned}
& \frac{\partial \hat{u}_{1}}{\partial \bar{x}}+\frac{\partial \hat{u}_{1}}{\partial \hat{y}}=0
\end{aligned}
$$



$$
\begin{aligned}
& y \frac{\partial \hat{T}_{1}}{\partial x}-\frac{1}{P r} \frac{\partial^{2} \hat{T}_{1}}{\partial \bar{y}^{2}}=\cdots \hat{u}_{0} \frac{\partial \hat{T}_{0}}{\partial \bar{x}^{2}}-\hat{v}_{0} \frac{\partial \hat{T}_{0}}{\partial \hat{y}},
\end{aligned}
$$

cte., with the following boundary conditions:

$$
\begin{aligned}
& \hat{u}_{n}=\hat{v}_{n}=0, \quad \hat{T}_{0}=\hat{T}_{w}(\ddot{x}) . \quad \hat{T}_{n}=0(n \neq 0), \quad \frac{\partial \hat{\tau}_{n}}{\partial \ddot{y}}=\left(1+\hat{T}_{n-1}\right) \frac{d \hat{p}_{n}}{d \tilde{x}} \quad \text { at } \quad \bar{y}=0, \\
& a_{n} \rightarrow 0, \quad \widehat{T}_{n} \rightarrow 0 \quad \text { for } \quad|\ddot{x}| \rightarrow \infty, \\
& \hat{u}_{n} \rightarrow \hat{A}_{n}+\int_{0}^{\infty} \hat{T}_{n}(\bar{x}, \bar{y}) d \bar{y}, \hat{T}_{n} \rightarrow 0 \text { for } \hat{y} \rightarrow \infty,
\end{aligned}
$$

and

$$
\hat{D}_{n}(\bar{x})=\frac{1}{\pi} \int_{-\infty}^{\infty} \frac{d \hat{A}_{n}\left(\tilde{x}_{1}\right) d \bar{x}_{1}}{d \bar{x}_{1}} .
$$

The leading-order solution can be oblaned using the Fourier transform defined for all variables as, for example,

$$
\tau_{1}(\bar{y})=\int_{\cdots, \psi_{2}}^{\alpha_{1}} \hat{\tau}(\bar{x}, \bar{y}) \exp \left(-i k_{r} \bar{x}\right) d \ddot{x}
$$

The tansformed equations are then given by

$$
\begin{aligned}
i k \bar{y} \tau_{O F} & =\frac{d^{2} \tau_{O Y}}{d \bar{y}^{2}}, \\
i k \bar{y} P_{1} \cdot T_{O Y} & =\frac{d^{2} \tau_{O !}}{d \bar{y}^{2}},
\end{aligned}
$$

with the following boundary conditions:

$$
\begin{aligned}
& T_{O F}(0)=\left.T_{w 1 ;} \quad \frac{d \tau_{O H}}{d \bar{y}}\right|_{y<0}=i k|k| \int_{0}^{w}\left(\tau_{O F}-T_{O H}\right) d y, \\
& \left.\tau_{01 \times}(\infty) \rightarrow 0, \quad T_{01:}(\infty)\right) \rightarrow 0,
\end{aligned}
$$

for any value of the wave number $k$. The first step is to obtain the solution up to the order of $\delta$. In this case the energy equation decouples from the condinuity and momentum equations. The solution to (22) is

$$
T_{O F}=T_{\mathrm{w} F:} \frac{\operatorname{Ai}\left[(i k P)^{1 / 3} \bar{y}\right]}{A i[0]}
$$


where Ai represents the Airy function. The solution to (21) with the approprate boundary conditions is



The pressure in Fourier space is given by

$$
p_{0 \mathrm{~F}}=\frac{T_{\mathrm{WF}}|k|(i k)^{1 / 3} A i^{\prime}[0]}{\operatorname{Pr}^{1 / 3} A i[0]\left(i k|k|-(i k)^{2 / 3} A i^{\prime}[0]\right)}
$$

Using the Fourier inversion theorem, we can obtain the pressure distribution, skin friction, and displacement thickness for any given surface-temperature function. The solutions of the linearized equations atre shown in the next section compared with the nonlinear solutions.

\section{Nonlinear Stearly Analysis}

For values of $T_{\mathrm{w}}$ not close to unity, the complete solution of the problem defined by the steady form of (12) (16) requires numerical ticatment. In tems of the fourier tansformed variables, defined in the previous section, the nondimensional nonlinear equations take the form

$$
\begin{aligned}
& i k u_{1}:+\frac{d u_{\mathrm{F}}}{d \mathrm{j}^{i}}=0 \text {, } \\
& i k \bar{y} \tau_{\mathrm{F}}-\frac{d^{2} \tau_{\mathrm{v}}}{d \hat{y}^{2}}=\cdots\left\{\hat{u} \frac{\partial \hat{\tau}}{\partial \bar{x}}\right\}_{F}-\left\{\hat{v} \frac{\partial \hat{\tau}}{\partial \bar{y}}\right\}_{F}-\left\{\frac{d \hat{p}}{d \bar{x}} \frac{d \hat{T}}{\partial \bar{y}}\right\}_{F},
\end{aligned}
$$

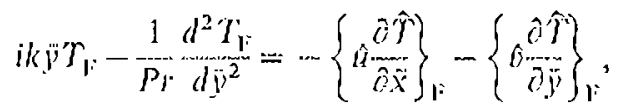

$$
\begin{aligned}
& u_{1}=\int_{0}^{y} \tau_{1:}(s) d s \\
& p_{1}=|k| \int_{0}^{\infty}\left(\tau_{1}(j) \cdots T_{\mathrm{V}}(j)\right) d j,
\end{aligned}
$$

with the boundary conditions

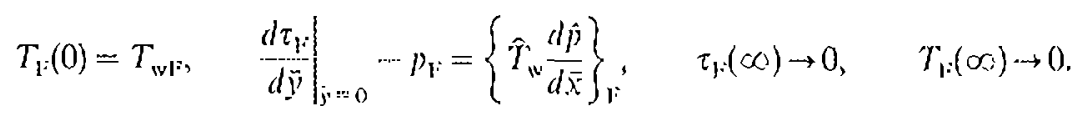

In the above cquations we have eliminated the transformed displacement function $A_{1}$ and have replaced it by the transformed version of the boundary condition

$$
A_{1}=\int_{0}^{\infty}\left[\tau_{1}(\bar{y})-T_{y}(\bar{y})\right] d \bar{y}
$$

The pressure, $p_{1}$, can also be suppressed by introducing (30) into the boundary condition (31).

We use a pseudospectral technique similar to that employed in [22] and [23]. We normalize the transversal coordinate for the lower deck in the following form:

$$
\zeta=\frac{\bar{y}}{1+\bar{y}} \text {. }
$$

The nonlinear terms are placed at the right-hand side of (26) (28). The procedure cmployed to initiate the calculations is to neglect these nonlinear terms and compute the fourier transformed vartables for the whole domain in $k$. The nonlinear terms are then inchded in the next iteration after evaluating the products in physical space, using results of the previous iteration and twice the fast fourier transform routine. We adid an artificial transiont term in the governing equations and use the "time" increment as a relaxation parameler. This procedure is repeated until convergence is achieved. The convergence criterion involved a tolerance of $10^{-5}$ in the pressure in the whole domain. Aliasing is removed using the $3 / 2$ rule [24]. We use 


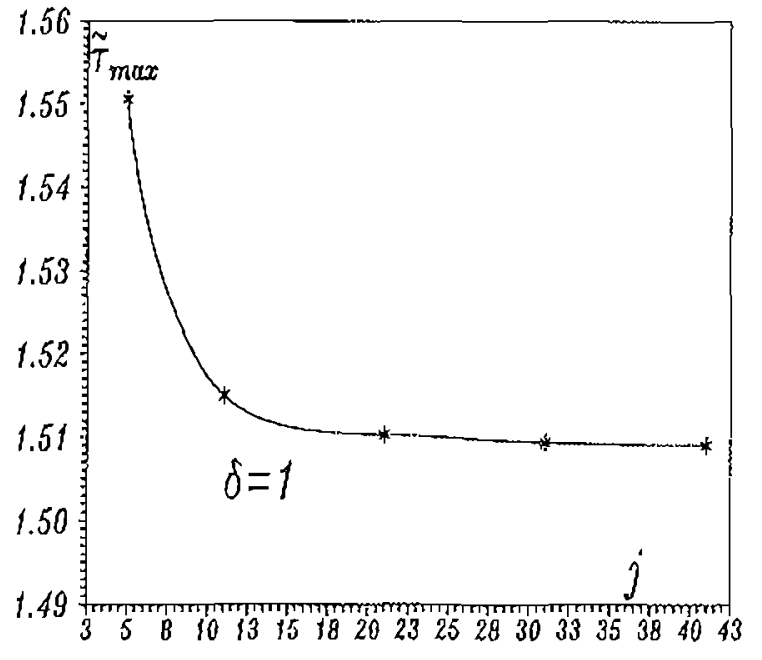

ligure 2. Inflence of the number of grid points in the transversal coordinate on the maximum value of skin friction for $\delta \ldots 1$.

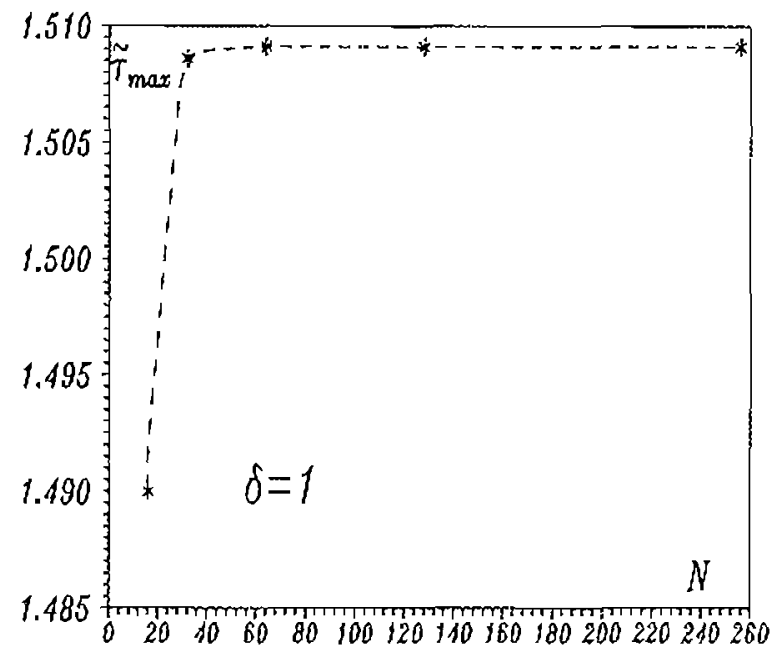

Figme. 3, Inlluence of the mumber of modes on the maximum vatue of skin fricion for $\delta=1$.

finite central differences to evaluate the derivatives in the left-hand side of (26) (31). The first derivative cvaluated at the surface for the boundary condition is discretized by second-order aceurale backward differences [22]. The integrals in (29) and (30) are approximated using the trapezoidal rule. We assume a beating function of the form

$$
\hat{T}_{w}=\frac{\delta}{1+\bar{x}^{2}}
$$

with $\delta$ now of order unity. The corresponding Fouricr transformed variable is then $T_{w \mathrm{~F}}=\pi \delta \exp (\cdots|k|)$. All mumerical computations of the nonlineas governing triple-deck cquations have been performed at the Cray-YMP computer of the National University of Mexico. The calculations presented here were made using a grid of $j=41$ points in the transversal direction and $N=256$ modes in the longitudinal coordinate, with the following endpoints $x_{j}= \pm 10 \pi$. ll gives a resolution in the Fourier space of $\Delta k=0.2$. This grid size was oblained after analyzing its influence on the resulling profiles using a heating parameter, $\delta=1$. 2 and 3 show this inlluence on the maximum value of the wall skin function, $\tilde{\tau}_{,} \tilde{\tau}_{\text {max }}$. In Figure 2 we vary the number of grid points in the transversal direction but maintain a constant number of modes, $N=256$. J1 can be secn that $\tau_{\text {max }}$ reaches almost a constant value of 1.5091 for $j>30$. On the other hand, in Figure 3 we show the infuence of the variation in the number of modes on $\bar{\tau}_{\text {max }}$, maintaining at constant number of grid points in the transversal dircction, $j=41$. Sixty four modes are cnough to reproduce with high accuracy the value of $\tilde{\tau}_{\operatorname{mix}}=1,5091$. Figure 4 shows the nondimensional pressure distribution, $\tilde{m}$, $\left.\mathrm{f}\right) \dot{\delta}=1$ for both the jinearized and the full nonlinear equations, using the 256 modes and a fluid with Prandt mumber unity. In fact what is plotted, at lexst for the linear case, is $\bar{p} P y^{1 / 3} / \delta$. Due to the gas expansion, there are two regions with adverse pressure gradients at the edges of the triple-deck middle zone, together with a favorable pressure-gradient region around the maximum plate temperature. The pressure first increases due to the expansion effects in the lower deck. Downstream the pressure decreases strongly reaching values lower than the ambient pressure, increasing again slowly asymptotically to the ambient value, for large positive values of $\bar{x}$. These adverse pressure gradients have a big in Ruence in regions close to the wall, where the convective terms are small. The nonlinear effects are rather small upstream of the maximum temperature point. However, there are two main effects due to the nonlinearities downstream. The peak of the minimum pressure is shifted further downstream and the underpressure itself is reduced. Figure 5 shows the nondimensional shear stress at the wall. For relatively large negative values of $\bar{x}$, the adverse pressure gradient produces a decreasing shear stress at the wall. The sceond adverse pressurengradient region (for positive values of $\bar{x}$ ) also produces a peak (stronger than the upstream one) in the minimum shear stress at the wall. The peak in the nondimensional wall shear stress, produced by the favorable pressure gradient close to $\bar{x}=0$, is much more pronounced than the two peaks in both adverse pressure regions. Thus, it is 


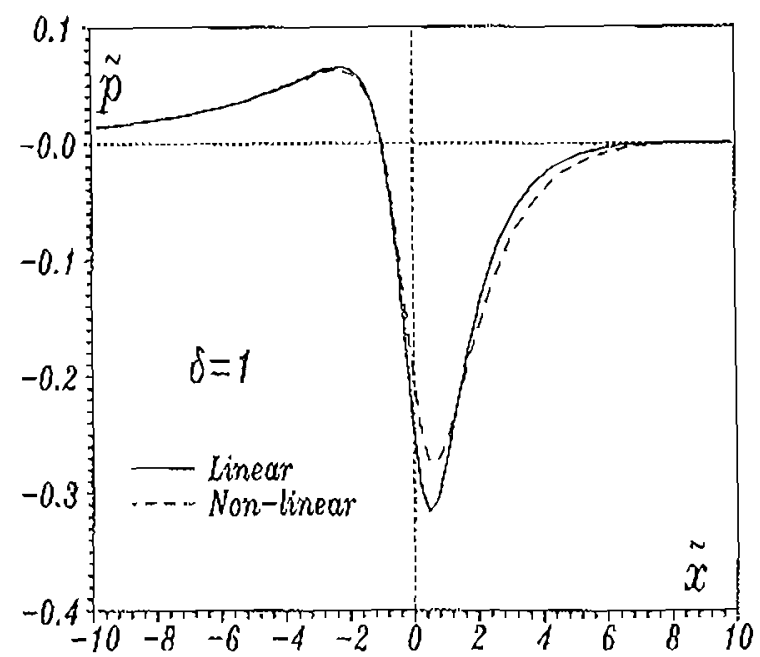

ligure 4. Nondimensional pressue destribution induced by a wall-temperature law given by $T^{*}=T_{w}^{*}\left[1+1 /\left(1+x^{* 2}\right)\right]$.



Jigure 5. Nondinensional skin-friction distribution induced by a wall-(emperature law given by $7^{4 *}=7^{*}\left[1+1 /\left(1+x^{* 2}\right)\right]$.

shown that cooling (instead of heating) will reduce the minimum wall shear stress for similar lemperature differences. The critical conditions for separation can be oblained $(\tilde{\tau}=0)$ from the lincar analysis, giving the extremely large value of $\delta_{\mathrm{c}} \approx 10 \mathrm{Pr}^{1 / 3}$. Practically, it is not possible to obtain separation using this smooth heating form. Figure 6 shows the values of the minimum values of the wall shear stress as a function of nondimensional temperature ratio $\delta$, for both the linear and nonlinear analyses. Surprisingly, there is not a big influence of the nonlinearities on that value, for values of $\delta \leqslant 4$. For values of $\delta$ smaller than 3 , the nonlinear effects tend to decrease this minimum wall shear stress. However, for larger values of $\delta$, the enect of the nonlincarties tries to reduce this value compared with the linear analysis. Therefore, separation will occur at larger values of $\delta$ as predicted by linear extrapolation. Figure 7 shows the maximum value of the wall shear stress as a function of $\delta$. The nonlincar effects always tend to increase this value.

The nondimensional pressure distribution and skin friction or wall shear stress for the cooling process are ploted in Figures 8 and 9, respectively, for the minimum possible value of the parameter $\delta, \delta=-1$. fior the linear case, the results are exactly the same, but with the sign changed, indicating the existence of a region with a strong adverse pressure gradient surrounded by two regions with moderately favorable pressure gradients. In this calse a high-pressure zone develops in the central region producing a strong

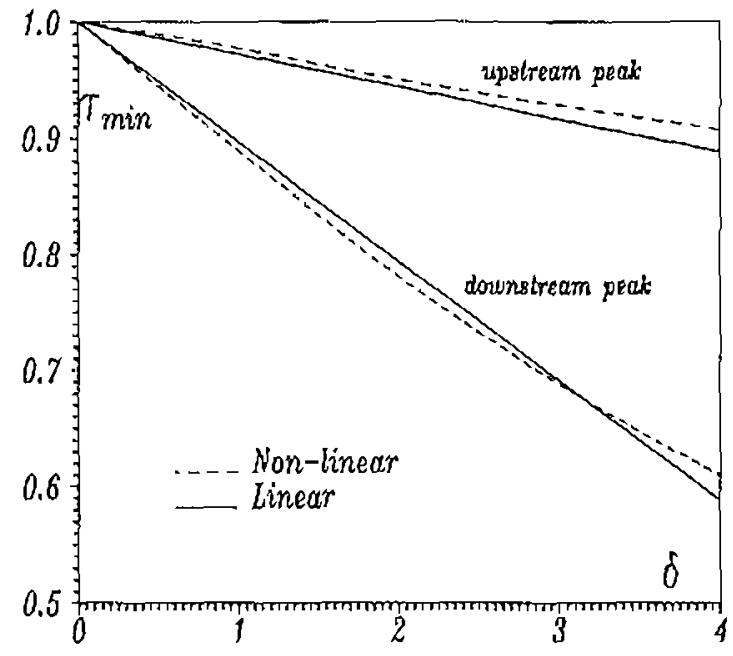

Jigure 6. Minimum skin-friction values as a function of the nondimensional temperature ratio $d$.

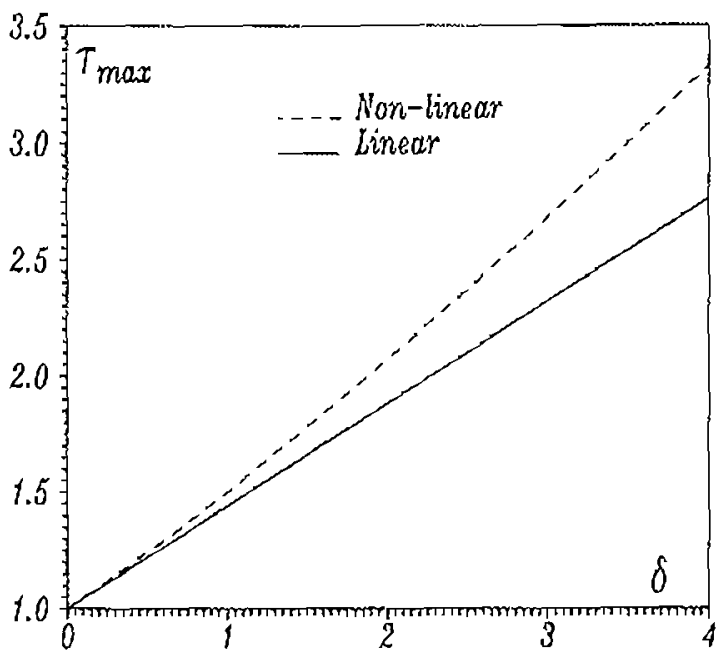

ligure 7. Maximun skin-friction values as a function of the rondimessional fomperatue atio $\delta$. 


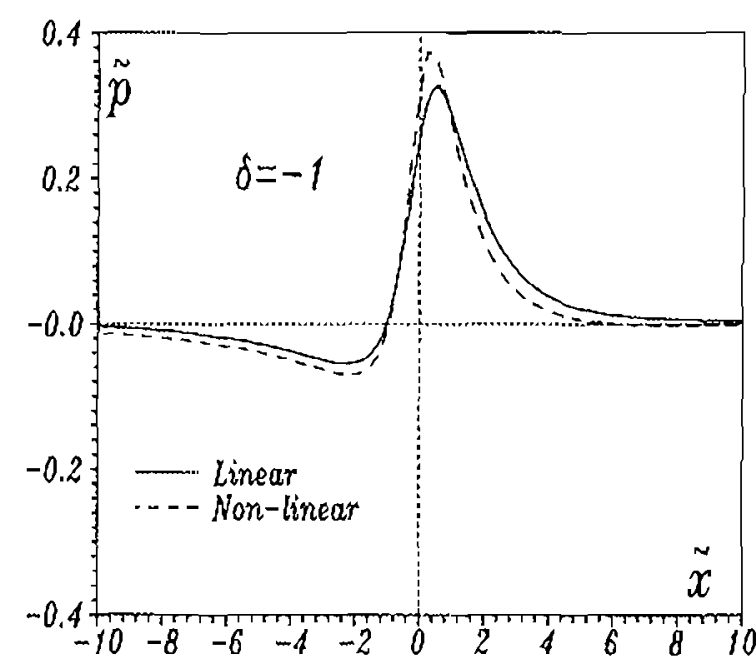

Figure 8. Nomdimensional pressure distribution induced by a wall-tomperature law given by $7_{*}^{*}=T^{*}\left[1 \cdots 1 /\left(1+x^{*}\right)\right]$.

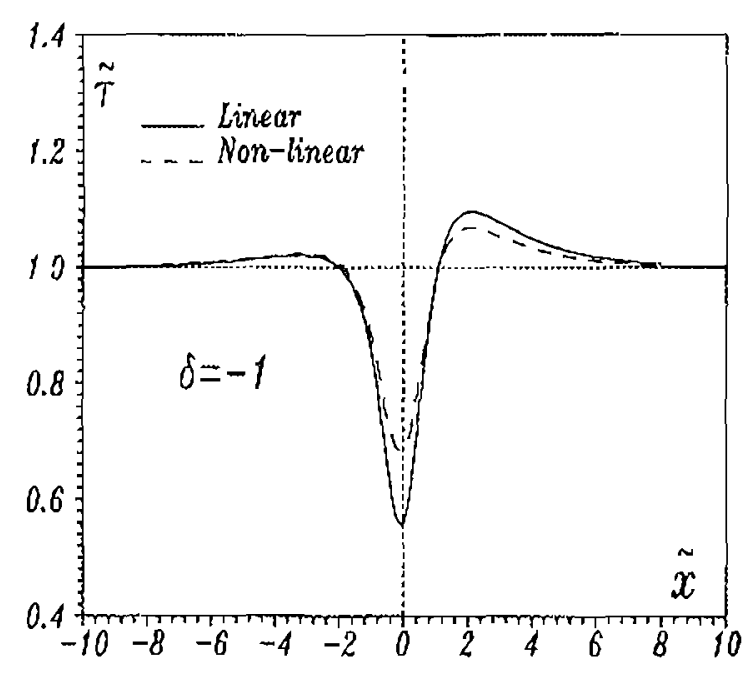

Figure 9. Nondinensional skin-friction distribulion induced b) a wall-temperaturc law given by $7^{*}=?_{*}^{*}\left[1-1 /\left(1+x^{*}\right)\right]$.

reduction in the minimum wall shear stress as indicated in those figures. However, separation cannot be achieved with the cooling process. Here, again, the nonlinear terms try to increase the minimum value of the wall shear stress.

Figures 10 and 11 show the nondimensional pressure distribution and wall shear stress for different posilive (heating) values of $\delta$. Incteasing the temperature ratio, both peaks, the minimum pressure and the minimum wall shear stress, are shifted downstream. We got convergence problems as we increased the value of $\delta$ further. We could not oblain a steady-state solution lor values of $\delta>4$, with the required convergence tolerance.

\section{Stability Analysis}

In this section we analyze the effect of a space-periodic surface thermal perturbation on lower branch boundary-layer stability, for high Reynolds numbers. To study this clfect on boundary-layer stability, we assume a small monochromatic thermal perturbation, of order $\delta$, with a given wave number $\beta$. In this case

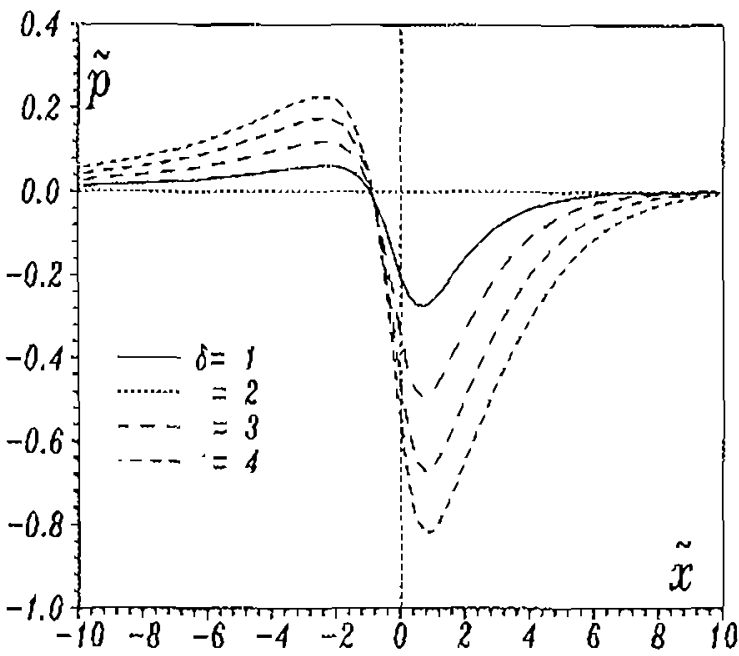

Fìgure 10. Nondimensional pressure distribution induced by at will-1emperatuic law given by $T^{*}=T^{*}\left[1+1 /\left(1+x^{* 2}\right)\right]$ for difierent values of $\delta$.

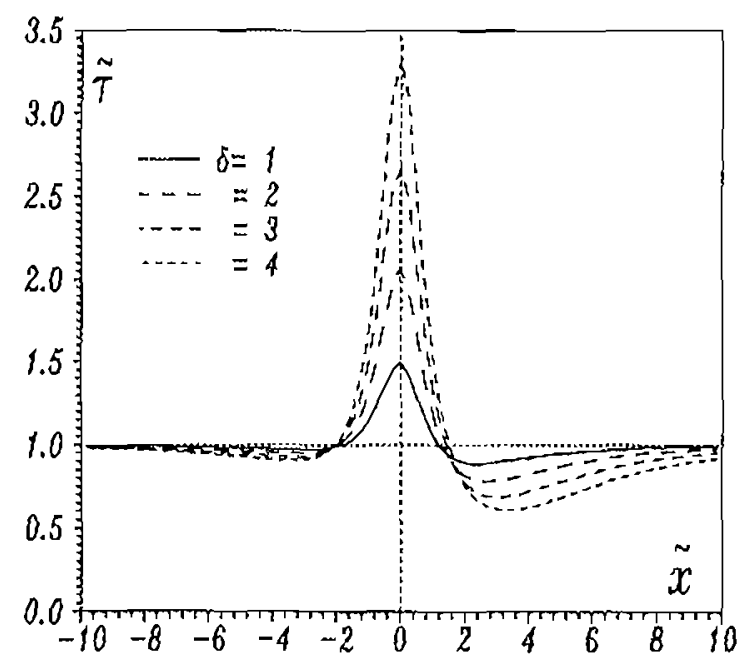

Jigure 11. Nondimensional skin-friction distribution induced by a wall-temperature law given by $\left.7^{\prime *}=\right\}^{*}\left[1+1 /\left(1+x^{* 2}\right)\right]$ for different values of $\delta$. 
we assume a solution of the form

$$
\begin{aligned}
& \hat{\tau}=\sum_{n=0}^{\infty} \delta^{n+1} \hat{t}_{n}(\tilde{x}, \bar{y})+\varepsilon \sum_{n=0}^{\infty} \delta^{n} \hat{\rho}_{n}(\bar{x}, \bar{y}, \bar{l}), \\
& \hat{T}=\sum_{n=0}^{\infty} \delta^{n}+1 \hat{T}_{n}(\tilde{x}, \bar{y})+\varepsilon \sum_{n=0}^{\infty} \delta^{n} \hat{o}_{n}(\bar{x}, \bar{y}, \bar{t}), \\
& \hat{p}=\sum_{n=0}^{\infty} \delta^{n+1} \hat{p}_{n}(\bar{x}, \bar{y})+\varepsilon \sum_{n=0}^{\infty} \delta^{n} \hat{\Pi}_{n}(\bar{x}, \bar{y}, \bar{l}),
\end{aligned}
$$

where $\delta \rightarrow 0$ and $\varepsilon / \delta \rightarrow 0$. Introducing thesc relationships into the triple-deck governing equations, we obtain, after collecting terms of the order 6 ;

$$
\frac{\partial \hat{\varphi}_{0}}{\partial \hat{T}}+j \frac{\partial \hat{i} \hat{\varphi}_{0}}{\partial \hat{\gamma}}-\frac{\partial^{2} \hat{\varphi}_{0}}{\partial \hat{y}^{2}}=0
$$

with the appropriate boundary conditions. We assume $\hat{\varphi}_{0}$ to be given by

$$
\hat{\varphi}_{0}=\sum_{k=0}^{\infty}\left\{F_{0 k}(\hat{y}) \exp \left[i\left(k \bar{x}-\left(D_{0 k} l\right)\right]+F_{o k}^{*}(\bar{y}) \operatorname{cxp}\left[-i\left(k \bar{x}-(\alpha)_{0 k}^{*} l\right)\right]\right\},\right.
$$

where $F_{0 k}^{*}$ and $\omega_{O k}^{*}$ denote the complex conjugate of the respective variable. $\omega_{0 k}$ is the first term of the expansion

$$
\omega_{k}=\sum_{n=0}^{\infty} \delta^{n}\left(\omega_{n k}\right.
$$

Equation (35) transforms to

$$
i\left(k \bar{y}-\omega_{O k}\right) F_{O k}=\frac{d^{2} F_{O k}}{d \bar{y}^{2}}
$$

with boundary conditions

$$
\left.\frac{d F_{0 k}}{d y}\right|_{y=0}=i k|k| \int_{0}^{\infty_{1}} r_{0 k} d \bar{j}, \quad \lim _{j \rightarrow \infty} \Gamma_{0 k}=0 .
$$

Equations (37) and (38) represent the perturbation on the incompressible boundary (lower branch), studied deeply by Smith and Burggraf [25], [26]. These equations reduce to the well-known dispersion relation

with

$$
A i^{\prime}(\gamma)=[i k]^{2 / 3}|k| \int_{x}^{\infty} \operatorname{si}(s) d s
$$

$$
x=-()_{0 k} \frac{(i k)^{1 / 3}}{k} .
$$

However, as mentioned by Smith [26], it is preferable to obtain the solution numerically (iteratively), giving an initial guess of $\omega_{0 k}$. This eigenvalue problem can be modified to the following boundary-value problem:

$$
i\left(k \bar{y}-\omega_{0 k}\right) f_{O K}=\frac{d^{2} f_{O k}}{d \bar{y}^{2}}
$$

with the boundary conditions

$$
\left.\frac{d f_{o k}}{d y}\right|_{y=0}=i k|k|, \quad \lim _{j \cdots \infty} f_{o k}=0
$$

and the restriclion

$$
\int_{0}^{\infty y} f_{0 k}(\bar{y}) d \tilde{y}=1
$$




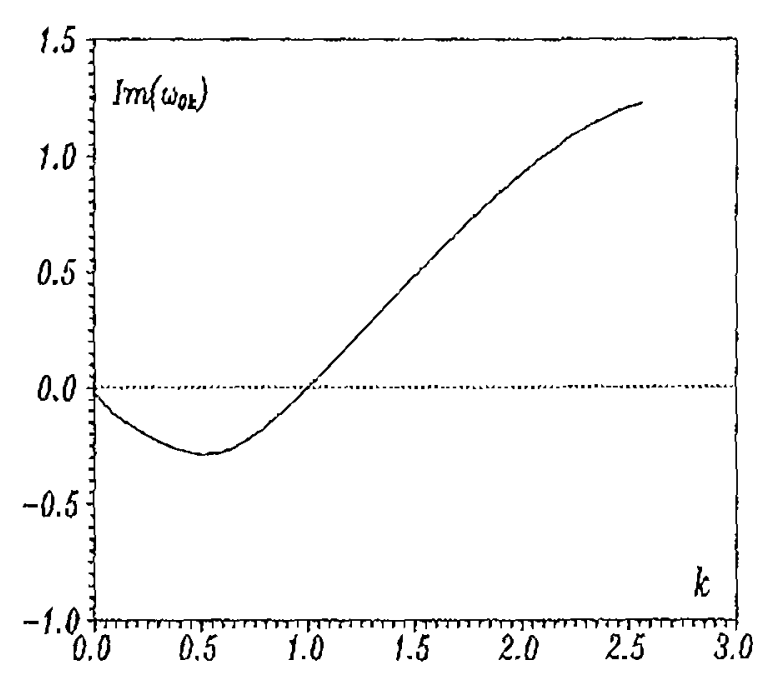

Iïgure 12. Lading-order value of the nondimensional groweh rate $\operatorname{Im}\left(\omega_{o x}\right)$ as a function of the ware number $k$.

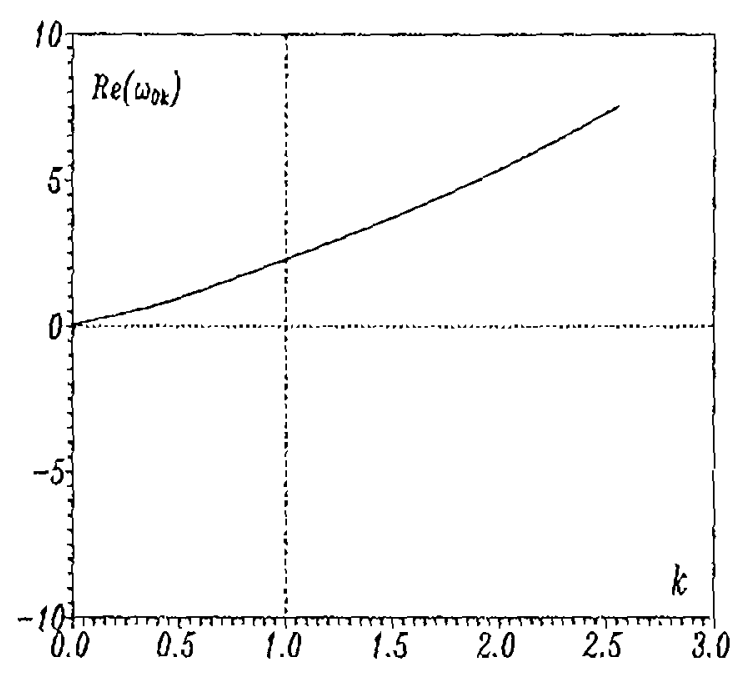

Jigure 13. Beading-opder value of the nondincusional ficquency Re(e) on as a fumction of the wate number $k$.

Equations (40) and (41) are numerically integrated giving an initial guess of the eigenvalues $\omega_{0 k}$. This value is corrected using the restriction condition (42) by the formula

$$
\omega_{o k}=k\left[|k|+\bar{y}_{\alpha}-\frac{i w_{0 \text { o. }}}{k u_{\text {ou. }}}\right] \text {, }
$$

where the velocity components

$$
u_{0 \alpha, k}=\int_{0}^{j y} F_{0 k}(j) d \tilde{y}, \quad v_{0 y, k}=\cdots i k \int_{0}^{j y} u_{0 x ; k}(\bar{y}) d \bar{y}
$$

are obtained in a previous jteration for a large fixed value of $\bar{y}_{,} \bar{y}_{\omega}$. This procedure is continued until convergence is achieved. Figures 12 and 13 show the results for the eigenvalue $\omega_{0 k}$ as a function of the wave number $k$. The boundary-layer flow is unstable for positive values of the imaginary part of $\omega_{0 k}$. We oblain the well-known values of neutral stability, for $k=k_{c}=1,0004 \ldots$ with a nondimensional frequency, real part of ()$_{0 x}=2.297 \ldots$. Pigure 14 shows the phase shift of both the nondimensional transversal velocity component in the main deck as well as the nondimensional vorticity at the wall, with the resulting pressure distribution, as a function of the wave number $k$. The nondimensional vorticity in the main deck is also plotted in the same figure. As the value of $k$ reaches the value of $k_{c}$, the transversal velocity component

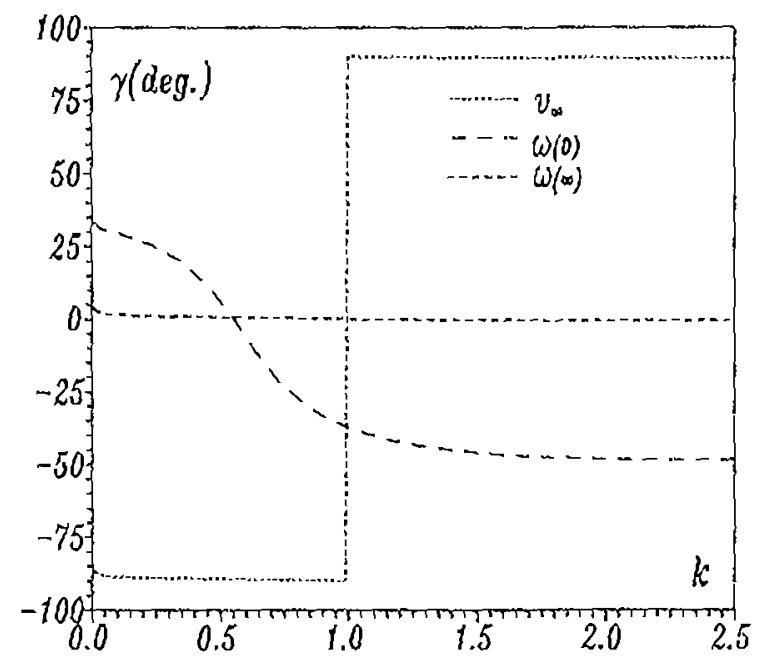

17igure 14. Phase shitt between pressure distribution and some now variables. 
changes in sign. In this case, for $k>k_{c}$, any pressure perturbation generales the conditions in the lower deck that induce a flow in the upper deck tending to increase such a perturbation. In order to study surface thermal effects on boundary-ayer stability, we have to go further by collecting terms of order $\varepsilon \delta$, giving

$$
\frac{\partial \hat{\varphi}_{1}}{\partial \bar{T}}+\bar{y} \frac{\partial \hat{\varphi}_{1}}{\partial \bar{x}}-\frac{\partial^{2} \hat{\varphi}_{1}}{\partial \bar{y}^{2}}=-U_{0} \frac{\partial \hat{\varphi}_{0}}{\partial \bar{x}}-u_{0} \frac{\partial \hat{\tau}_{0}}{\partial \bar{x}}-V_{0} \frac{\partial \hat{\varphi}_{0}}{\partial \bar{j}} \cdots v_{0} \frac{\partial \hat{\tau}_{0}}{\partial \dot{y}}-\frac{\partial \hat{\Pi}_{0}}{\partial \bar{x}} \frac{\partial \hat{T}_{0}}{\partial \bar{y}}-\frac{\omega_{1 k}}{\omega_{0 k}} \frac{\partial \hat{\varphi}_{0}}{\partial t}
$$

where

$$
U_{0}=\int_{0}^{\infty} \hat{\tau}_{0} d \bar{y}, \quad V_{0}=-\int_{0}^{\infty} \frac{\partial U_{0}}{\partial \bar{x}} d \bar{y}, \quad u_{0}=\int_{0}^{\infty} \hat{\rho}_{0} d \bar{y}, \quad v_{0}=-\int_{0}^{\infty} \frac{\partial u_{0}}{\partial \bar{x}} d \bar{y} .
$$

In Fourier transform space, (45) lakes the form

$$
\begin{aligned}
& i\left(k \bar{y}-(1)_{0 k}\right) F_{1 k}-\frac{d^{2} F_{1 k}}{d \bar{y}^{2}}=i \omega_{1 k} F_{0 k}-i\left[U_{0 \beta} F_{0(k \beta)}(k-\beta)+U_{0\}}^{*} F_{0(k \cdot s)}(k+\beta)\right] \\
& -i \beta\left[u_{O(k-\beta)} \hat{\tau}_{O \beta}+u_{O(k+\beta)} \hat{\tau}_{O \beta}^{*}\right]-\left[V_{O \beta} \frac{d F_{O(k \cdots \beta)}}{d \bar{y}}+V_{0 \beta \beta}^{*} \frac{d F_{O(k+\beta)}}{d \bar{y}}\right]
\end{aligned}
$$

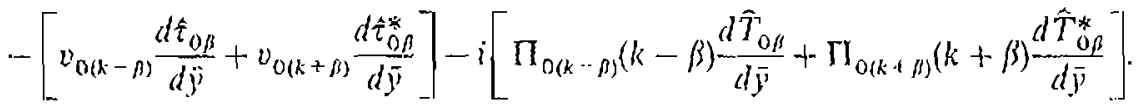

The monochromatic temperature perturbation can be written as

$$
T_{w^{\prime}}=\frac{\delta}{2}\{\exp [i(\beta \bar{x}+\alpha)]-\exp [-i(\beta \bar{x}+\alpha)]\},
$$

where $\alpha$ is the phase shift. Up to first order in $\delta$, the solution in Fourier space is given by

$$
\begin{aligned}
& \hat{T}_{0 \beta}=\frac{\exp (i \alpha) A i\left[(i \beta P r)^{1 / 3} \bar{j}\right]}{2 A i[0]}, \\
& \hat{\tau}_{O \beta}=\frac{\exp (i \alpha) i \beta|\beta| A i\left[(i \beta)^{2 / 3} \ddot{y}\right]}{2 \beta r^{1 / 3} A i[0]\left(i \beta|\beta|-(i \beta)^{2 / 3} A i^{1}[0]\right)^{\prime}} \\
& \hat{B}_{O \beta}=\frac{\operatorname{cxp}(i c)|\beta|(i \beta)^{1 / 3} A i^{\prime}[0]}{2 P^{1 / 3} A i[0]\left(i \beta|\beta| \cdots(i \beta)^{2 / 3} A i^{\prime}[0]\right)} \text {. }
\end{aligned}
$$

In (49) - (51) addition of the complex conjugate is implied. The solvability condition gives the first-order correction for the complex frequency perturbation, $(1)_{1 k}$, which measures the effect of surface thermal perturbation on boundary-layes stability. This valke can be found after solving the adjoint problem defined by

$$
-i\left(k \ddot{y}-\omega \omega_{0 k}^{*}\right) G_{k} \cdots \frac{d^{2} G_{k}}{d \bar{y}^{2}}=i k|k| G_{k}(0)
$$

with boundary conditions

$$
\left.\frac{d G_{k}}{d \bar{y}}\right|_{y: 0}=0, \quad \lim _{i \rightarrow \infty} G_{k}(\bar{y})=0
$$

In fact, the asymptotic behavior for large values of $\bar{y}$ is given by

$$
\frac{G_{k}}{G_{k}(0)} \sim-\frac{|k|}{\left(\bar{y}-\left(\omega_{0 k}^{*} / k\right)\right.}+\frac{2|k|}{k\left(\bar{y}-\omega_{0 k}^{*} / k\right)^{4}}+\cdots .
$$

Figures 15-17 show the first-order correction for the growth rate, $\operatorname{Im}\left(\omega_{1 k}\right)$, for all leading-order stable wave numbers $k\left(k<k_{c}\right)$ and different values of the thermal perturbation wave number $\beta$. All possible phase shifts 


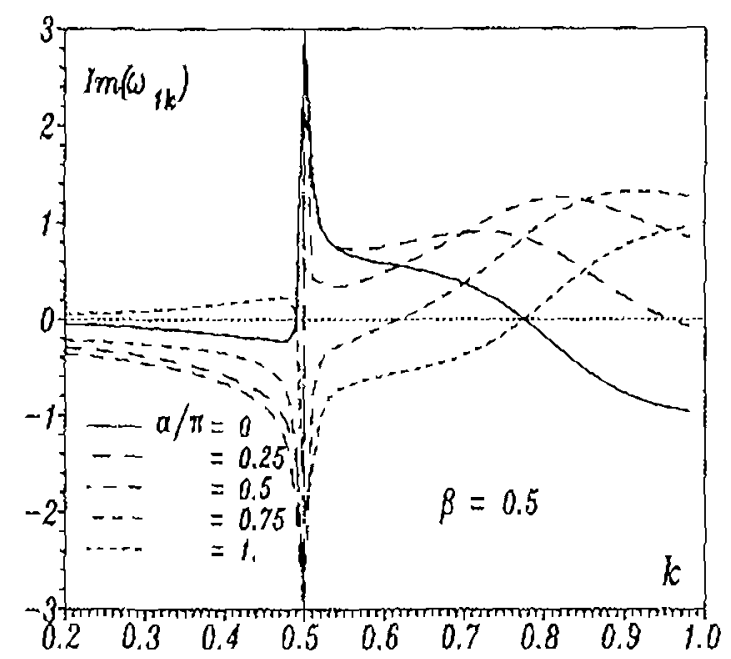

Pigure 15. First-order vahe of the mondimensional growth rate as a function of the wave number $k$ for $\beta=0.5$.

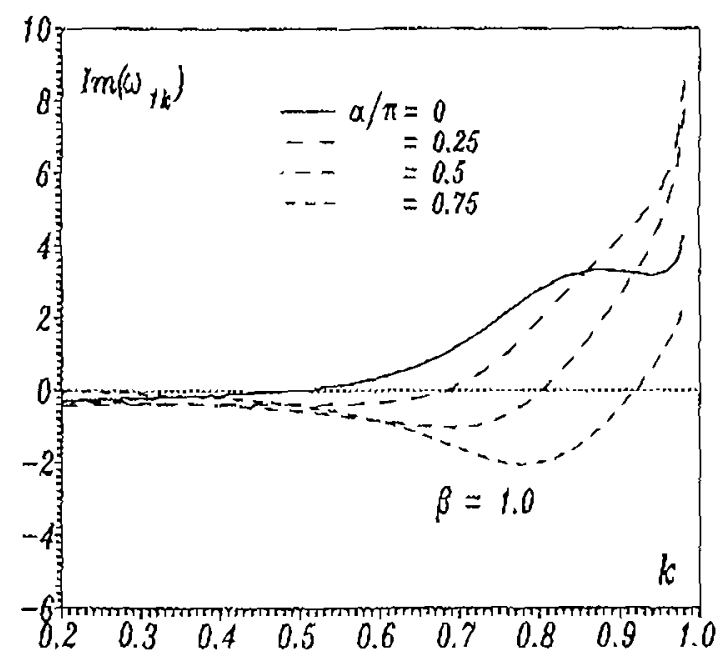

liggtre 16. Hirst-order value of the nondimensional growth rate as a function of the wate number $k$ for $\beta \Rightarrow 1$.

are scanned in the upper half-plane $0<\alpha<\pi$. The corresponding phase shifts to the lower half-plane can be obtained using the fact that $\operatorname{Im}\left[\omega_{1 k}(\alpha)\right]=-\operatorname{lm}\left[\omega_{1 k}(-\alpha)\right]$. Due to this, the important variable is the absolute value of the first-order correction of the cigenvalue, $\omega_{1 k}$. In all three figures we can see how monochromatic themal perturbation, with amplitude $\delta$, modifies boundary-layer neutral stability, causing all wave numbers to be unstable, depending on the value of $\delta$. The same results ate shown in Figures 18 and 19 for $\omega_{1 k}$ as a function of the wave number of the thermal perturbation $\beta$, for a given fixed value of $k$. 'The shift of the neutral stability curve, which depends not only on the parameter $\delta$, but also on the perturbation wave number $\beta$, can be oblained up to first order from the relationship

$$
\operatorname{Im}\left[\omega_{0 k}\right]+\delta_{r k}\left\{\max \left(\ln [\omega)_{1 k}(\alpha, \beta)\right]\right\}=0,
$$

where $\delta_{c k}(\beta)$ corresponds to the tenperature ratio which makes any perturbation of wave number $k$ unstable. The biggest influcnce occurs for $k=\beta$, resulting in a resonant-lype interaction which produccs the singularity in the figures. Figure 20 shows $\delta_{c k}$ as a function of $k$ for different values of the thermal

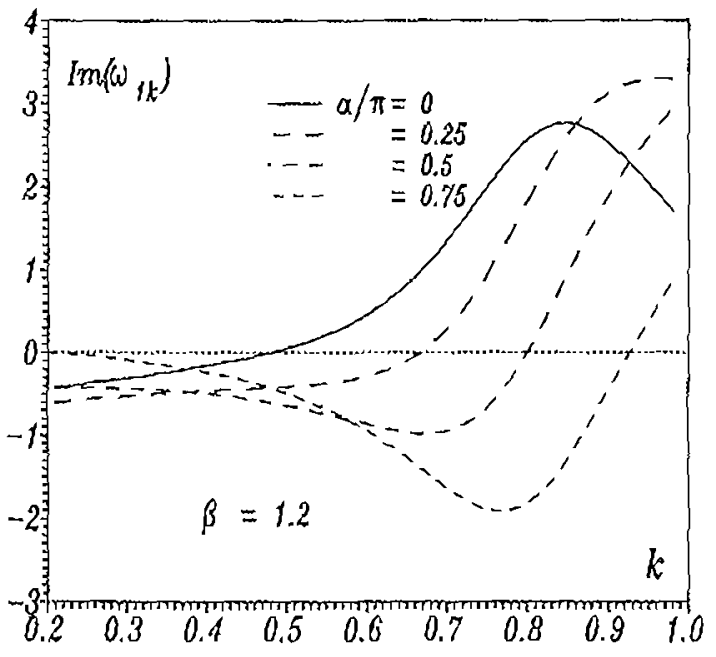

Jigure 17. First-egdes value of fle nondimensional groweth rate as a function of the wave number $k$ for $\beta=1.2$.

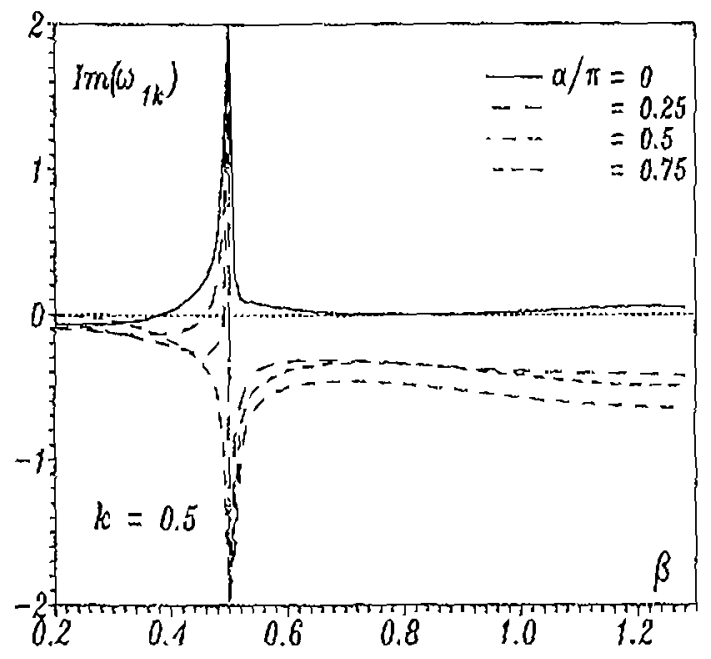

Irigure 18. First-order value of the nondincmsional growth rate as a function of the perturbation wave munber $\beta$ for $k=0.5$. 


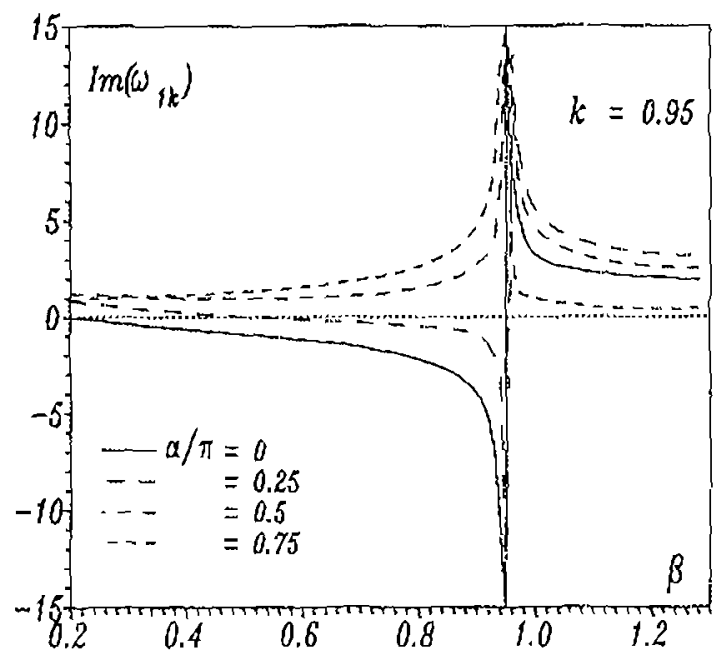

Kigure 19. First-order value of the nondimensional growth rate as a function of the perturbation wave muber $\beta$ for $k=0.95$.

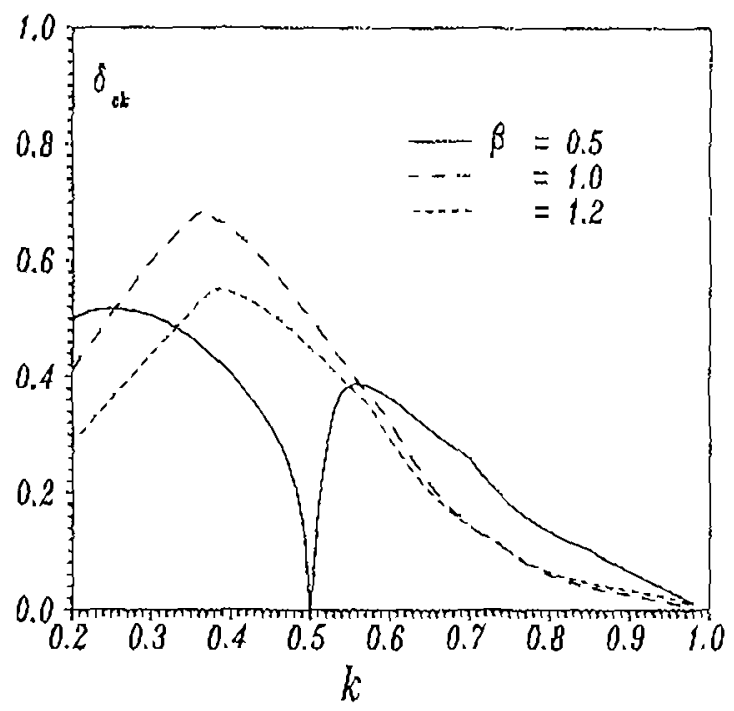

Figure 20. Ciritical value of the thermal stuface infersity $\delta$ as a function of the wavenumber $k$ for dificrent values of $\beta$.

perturbation wave number $\beta$. Relatively small values of $\delta$ are needed to make, up to first order; the boundary-layer fow unstable for all wave numbers.

\section{Concluling Remarks}

The structure and stability of a gascous boundary-layer flow perturbed by thermal effects at the body surface, was analyzed in this work using the formalism of riple-deck theory. The nonconstant density unsteady governing equations are reduced to the quasi-incompressible case by using the HowarthDorodnitzyn transformation for an ideal gas with the Chapman-Rubesin palameter of unity. The linear and nonlinear steady triple-dock governing cquations have been numerically integrated using spectral numerical methods. Both heating and cooling were analyzed using a smooth surface-temperature distribution given by $T_{\mathrm{w}}^{*}=T_{*}^{*}\left[1+\delta /\left(1+x^{* 2}\right)\right]$. For the values of the heating parameter $\delta$ considered, $-1<\delta<4$, it was not possible to obtain boundary-layer separation for the smooth surface-temperature profite assumed. For larger values of $\delta$ it was not possible to obtain a steady-state solution with the appropriate convergence toletance. The heating profile studied in Fouricr space is $\pi \delta \exp (\cdots|\tilde{x}|)$, which clearly contains unstable modes. Using lincar analysis, the critical conditions for boundary-layer separation give the extremely large value of $\delta_{c r}=10 \mathrm{Pr} \cdot 1 / 3$. Practically, it is impossible to get boundary-layer separation using this smooth profile, contrary to that obtajned using a step change in surface temperature [6]. For the same values of $\mid \delta$, cooling reduces in a stronger way lhe minimum wall shear stress, but without any possibility of separation. Using perturbation methods, we have obtained fist-order correction of the lower branch neutral stability curve for the boundary-layer now. This correction is produced by a low-intensity monochromatic surface thermal perturbation, in a gaseous fow. The shift of the neutral stability is then computed for different values of the thermal perturbation wave number $\beta$. We oblained both quantitatively and qualiatively the effect of wall themal perturbation on the stability of the boundary-layer flow, making unstable some otherwise stable modes, depending on the temperatue ratio.

\section{References}

[1] K. Stewartson and P.G. Williams, Self-induced separation, Proc. Roy. Soc. London Ser. A, vol. 312, p2. $181-206(1969)$.

[2] A.J: Messiter, Boundary layer flow near the trailing edge of a llat plate, S1AM J. App. Math, vol. 18, p3. 241 -257 (1970).

[3] K. Stewartson, D'Almbert's paradox, SIAM Rati, vol. 23, pp. $308-343$ (1981).

[4] I:T. Snith, On the high Reynolds number theory or haminat fows, J. Appt. Math, vol. 28, pp. 207 -281 (1982). 
A.F. Messites and A. Linith, The vertical hat plate in lamina free convection: elfects of leading and traing edges and discontinuous temperature, $Z$. Angew. Math. Physe, vol. 27, Pp. 633 - 651 (1976).

1. Ménde\%, C. Treviño, and $\wedge$. Liñin, Boundary layer separation by a slep in surface temperature, Lernat. I. Heut Mass Trunsf, vol. 35 , pp. 2725 -2738 (1992).

F.T. Smib, On the non-parallel boundary layer stability of the IBhsius boundary layer, Proe. Roj. Sor. Lomdon Ser. A, vol. 366, pp. $91-109(1079)$.

1"'T. Smith, Nort-lincar stability of boundary byers for disturbances of various sizes, Proc. Roy. Soc London Ser. A, vol. 368 pp. 573589 (1979).

F.T. Smill, Proc. Roy. Sor. London Ser. A, vol. 371, p. 439 (1980).

M.E. Goldstein, Scatcring of acoustic waves into Tolmien-Schlichting waves by small streamwise viliations in surface gecomcly, J. Fhidel Mech, vol. 154, p. 509 (1985).

M.IE. Goldstein and L.S. Hultgren. A note on the generation of Tollmein- Sellichting waves by sudden surface-curvature change, .I. Fluid Mech., vol. 181, p. 519 (1987).

P. l.eehcy and $P$. Shapiro, l.cading edge efiect in Luminar boundary layer excitation by sound, in Lominar-Turbutent Transition (cd. R. Eppler and H. Fasel), Springer-Verlag, New York, pl). 321 -33i (1979).

R.J. Bodonyi, W.J.C. Welch, P.W. Duck, and M. Tadjfar, $\Lambda$ numerical study of the interaction between unsteady free-strenm disturbances and locklized vatiations in surlace geometry, I. Fitrid Mech, vol. 209, p. 285 (1989).

S.O. Secdongui, R.1. Bowles, and F.T. Smith, Surface-cooling elfects on compressible boundary-layer instability, and on upstream influence, Lewopect J. Mech. B/F"hids, vol, 10, p. 117 (1991).

V.I. Lysenko and A.A. Maslov, The effect of cooling on supcrsonic boundary-layer stability, J. Fitud Mech, vol. 147, p. 39 (1984).

S.N. Brown, H.K. Cheng, and C.J. I.ce, Inviscid-viscous interaction on triple-deck scalcs in a hypersonic flow with strong wall cooling, J. Fhid Mech., vol, 220, p. 309 (1990).

D.l..'Turcolte, Stable combustion of a high velocity gas in a heated boundary layer, J. Aeronaut. Sci, vol. 27, pp. 509--516(1960)

$T$. Hirano and Y. Kamo, Aerodynamical and thermal structures of a lamina boundary layer over a flat plate with a diffusion name, Proc. XIV Imernat. Sump. on Combustion, p. 391 (1973)

C. Treviño, W. Stüttgen, and N. Pelers, Pressure gradients due to gas expansion and gasification effects in a boundary layer combustion of a condensed fucl, W'ärme Stoffïbertragl., vol. 25, pl). 309 319, (1990).

C. Trevino, W. Stültgen, and N. Peters, Higher order efects in the premixed boundary kyer combustion, J. Propul. Power, vol. 6, no. 3, pp. 237242 (1990).

L. Irowarh, Conceming the effect of compressibitiry on laminar boundary layers and their separation, Proc. Roy. Soce lowdon Sel. A, vol. 194, p. 16 (1948)

P.W. Duck and O.R. Burggraf, Spectral solutions for threedimensional triple-deck flow over surace topography, J. Finid Mech., vol. 162, pp. 122 (1986).

O.R. Burggraf and P.W. Duck, Spectual computation of tripledeck fows, in Numerical and Physical Aspects of Acrodymanne Flows (ed. T. Cebeci), Springer-Verlag, New York (1981).

C. Camuto, M.Y. Hussaini, A. Quarteroni and T.A. Zang, Spectral Mothods in Fhud Dynamics, Springer-Verlag, New York $(1988)$.

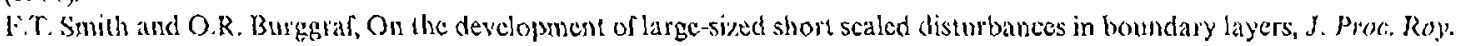
Soc. London Ser. A, vol. 399, p. 25 (1985)

F.T. Smith, On the first mode instability in subsonic, supersonic or hypersonic boundary layers, J. Fhid Mech., vol. 198. [p). $127 \cdot 153(1989)$. 